\title{
Optical Light Curve of Nova KT Eridani
}

\author{
W. P. Chen ${ }^{1}$, L. W. Hung ${ }^{1}$ and Fred Walter ${ }^{3}$ \\ ${ }^{1}$ Graduate Institute of Astronomy, National Central University, Taiwan \\ email: wchen@astro.ncu.edu.tw \\ ${ }^{2}$ Dept of Physics, National Central University, Taiwan \\ ${ }^{3}$ Dept. of Physics and Astronomy, Stony Brook University, USA
}

\begin{abstract}
We present the optical light curve of the very fast nova, KT Eridani, from its outburst in November 2009 to quiescence in 2012. Comparison of our data with the 24-hour monitoring by Swift on 2010 March 31 indicates a possible anticorrelation between the X-ray and optical emissions. A period of $57 \mathrm{~d}$ is found in the long-term optical light curve, which we interpret as the orbital modulation of the hot spot on the white dwarf disk accreting material from an early K-type giant. Our study lends support to the notation that KT Eri may be a recurrent nova.
\end{abstract}

Keywords. Accretion disks, binaries: close, novae, white dwarfs, X-rays: binaries

\section{Introduction}

Nova KT Eridani 2009 (RA=04:47:54, DEC $=-10: 10: 43$, J2000) was detected by Itagaki (2009) at a unfiltered brightness of $8.1 \mathrm{mag}$, in contrast to the quiescent $15 \mathrm{mag}$, on 25.536 UT November 2009. It was later found to have a peak visual brightness of 5.4 mag at 14.632 UT November 2009, i.e., it brightened 3 mag in $1.6 \mathrm{~d}$, declined 2 mag with a time scale of $t_{2}=6.6 \mathrm{~d}$ and 3 mag with $t_{3}=13.6 \mathrm{~d}$, making KT Eri a very fast nova. It was found to be a super-soft X-ray (Bode et al. 2009) and a radio source (O'Brien et al. 2010) with many characteristics of a recurrent nova (Beardmore et al. 2010, Bode et al. 2010).

Hung et al. (2011) reported the optical light curve of KT Eri up to the early 2011, with data collected with the $1 \mathrm{~m}$ at Lulin (Taiwan), the $0.8 \mathrm{~m}$ at Tenagra (Arizona, USA), and the SMARTS $1.3 \mathrm{~m}$ at CTIO (Chile). In particular, optical monitoring was carried out together with a SWIFT 24-hour campaign on 31 March 2010. The X-ray flux was clearly variable and seemed to anticorrelated with the optical flux at least for some duration.

Figure 1 shows the optical V-band light curve observed with the SMARTS $1.3 \mathrm{~m}$, from the outburst to when the nova returned to quiescence with $\sim 15$ mag in mid-2012. The quiescent light curve confirmed the $\sim 57 \mathrm{~d}$ period previously suspected during the decline phase (Hung et al. 2011).

As presented in Hung et al. (2011), the $57 \mathrm{~d}$ period could not have been the orbital period of the binary, because the periodicity was evidenced in the B band (for which the companion should not contribute much light), but not in the $\mathrm{V}$ band soon after the outburst, indicative of a hot source. Instead, the period should be due to the orbital motion of the hot spot on the accretion disk, which is orbiting the white dwarf - not in Keplerian motion, but synchronized with the companion (donor). With an assumed combined binary mass of $2.5 \mathrm{M}_{\odot}$, a separation of $0.39 \mathrm{AU}$ was derived between the secondary and the white dwarf. With this distance, a main-sequence companion would have too small a size to fill its Roche lobe. The companion must be a giant or a supergiant. Therefore, KTEri has characteristics, i.e., with a giant secondary and a long orbital period, similar to those of a recurrent nova. 


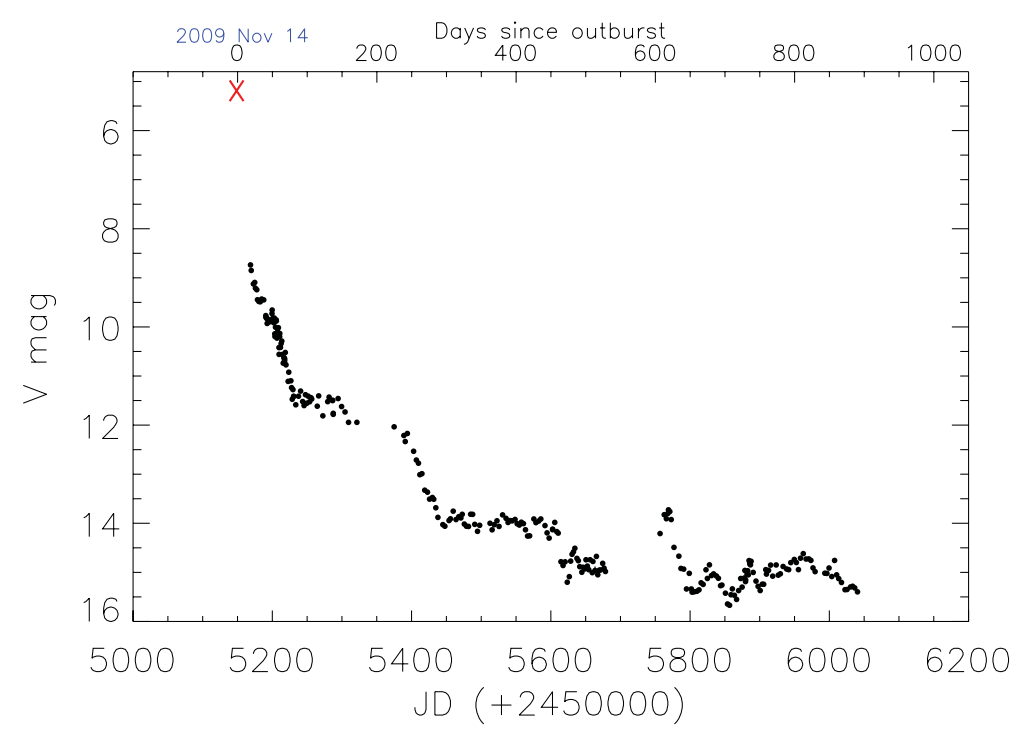

Figure 1. Optical light curve of KT Eridan since the outburst on 14 November 2009.

The size of the Roche lobe of the secondary is estimated by the approximate formula (Paczynski 1971), $r_{1} / A=0.46224\left(M_{1} /\left(M_{1}+M_{2}\right)^{1 / 3}\right.$, where $r_{1}$ is, with the spherical approximation, the radius of the lobe around mass $M_{1}$, and $A$ is the semi-major axis of the binary orbit. Using an $M_{1}=1.3 \mathrm{M}_{\odot}$ white dwarf, an $M_{2}=1.2 \mathrm{M}_{\odot}$ giant (the result depends only weakly on the mass), and $A=0.39 \mathrm{AU}$, we get $r_{1}=0.14 \mathrm{AU}$, or $29 \mathrm{R}_{\odot}$, for the size of the secondary. This is about the size of an early K giant (Cox 2000; Dyck et al. 1996). At a distance of $6.5 \mathrm{kpc}$ and a reddening of $E(B-V)=0.08 \mathrm{mag}$ (Ragan et al. 2009), a quiescence $m_{V}=15$ mag yields an absolute magnitude of $M_{V}=0.7$ mag, consistent with being an early K giant (Cox 2000). The pre-nova 2MASS colors of KT Eri, $J-H=0.46 \mathrm{mag}$ and $H-K s=0.07 \mathrm{mag}$, also suggest a late $\mathrm{G}$ giant (Bessell \& Brett 1988). Extended spectra and photometry of a collected sample of novae observed with SMARTS, including KT Eri, can be found in Walter et al. (2012).

\section{References}

Beardmore, A. P., et al. 2010, ATel, 2423

Bessell, M. S. \& Brett, J. M. 1988, PASP, 100, 1134

Bode, M. F., et al. 2010, ATel, 2392

Cox, A. N. 2000, Allen's Astrophysical Quantities (4th ed.; AIP Press, Spinger, New York)

Dyck, M., Benson, J. A., Van Belle, G. T., \& Ridgway, S. T. 1996, AJ, 111, 1705

Hounsell, R., et al. 2010, ApJ, 724, 480

Hung, L. W., Chen, W. P., \& Walter, F. M. 2011, ASPC, 451, 271

Itagaki, K. 2009, CBET, 2050

Orio M., Covington J. \& Ogelman H. 2001, A\&A, 373, 542

O'Brien T. J., et al. 2010, ATel, 2434

Paczynski, B. 1971, ARAA, 9, 183

Payne-Gaposchkin C. 1957, The Galactic Novae (Amsterdam: North-Holland)

Ragan E., et al. 2009, ATel, 2327

Walter, F. M., Battisti, A., Towers, S. E., Bond, H. E \& Stringfellow, G. S. 2012, eprint arXiv:1209.1583

Yamaoka, H., et al. 2009, IAU Circ., 9098, 1 